\title{
Active control of the surface potential of nanostructured layers
}

\author{
M. Dominguez-Pumar ${ }^{1}$, L. Kowalski ${ }^{1}$, E. Llobet $^{2}$ and R. Calavia ${ }^{2}$
}

\begin{abstract}
The objective of this paper is to show the first results obtained with a control designed to keep constant the average surface potential of a nanostructured layer. This condition is equivalent to keeping constant the resistivity of the layer measured at a constant reference temperature. The proposed closedloop control achieves this objective by adequately changing the average temperature of the temperature waveforms applied to the nanostructured layer. Experiments are shown on which the control is applied to a layer made of Au-functionalized $\mathbf{W O}_{3}$ nanoneedles.
\end{abstract}

\section{INTRODUCTION}

Active layers based on nanostructures are used in many areas such as biosensors or gas sensors. The high surfaceto-volume ratios for nanomaterials such as nanoparticles, nanotubes or nanoneedles make them excellent candidates for their use in the detection of biomolecules or gases, [1]. In the case of gas sensors, variations of the surface potential the surface potential of the sensing layer are monitored by measuring its resistivity, [2]. In the case of silicon nanowires or carbon nanotubes FETs (SiNW-FET and CNT-FET respectively) changes in the surface potential of the semiconductor channel generate changes in the channel conductivity of the transistor, [3].

The conductivity of nanostructured layers responds in most cases to the potential barrier theory, and therefore can be understood as a two-time scale dynamic system. Changes in temperature generate changes in the conductivity of the layer through two mechanisms:

- a first mechanism by which the redistribution in energies of the charge carriers generate fast changes in the conductivity of the layer, and

- a second mechanism by which changes in the chemical reactions rates generate slow changes in the conductivity.

Keeping constant the temperature of the layer ensures that changes in the conductivity are only due to changes in the chemical reaction rates taking place in the layer. The approach in this paper is the design of a control loop scheme to enforce a constant conductivity of the layer, measured at a constant reference temperature, by generating temperature profiles with the adequate average temperature. These techniques are often

\footnotetext{
1 Micro and Nano Technologies Group, Electronic Engineering Department, Universitat Politècnica de Catalunya, Spain

${ }^{2}$ MINOS-EMaS, Department d'Enginyeria Electrónica, Universitat Rovira i Virigili, Tarragona, Spain

We are indebted to Dr. Annanouch for growing the tungsten oxide nanoneedles on the micromachined substrates provided by Dr. Cané and Dr. Gracia Funded in part by MINECO under grants num. TEC2013- 48102-C2-1-P and TEC2012-32420. E.L. is supported by the Catalan Institution for Research and Advanced Studies via the ICREA Academia award.
}

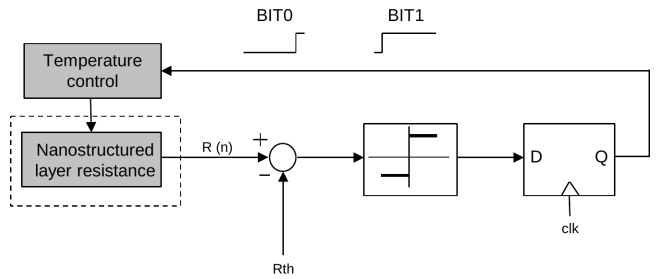

Figure 1. Closed-loop control of surface potential. At each sampling time, depending on whether the chemical resistance, measured at the reference temperature $T_{\text {high }}$, is below (above) the desired value, a BIT1 (BIT0) temperature waveform is applied to the sensor.

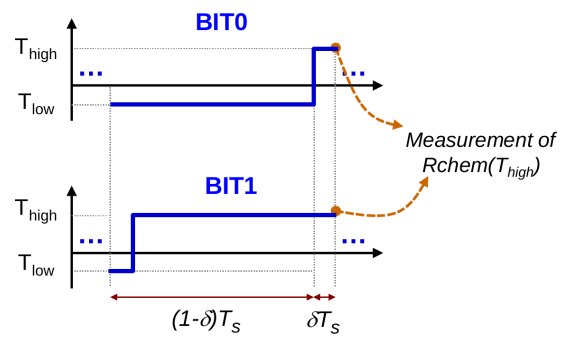

Figure 2. Temperature waveforms applied during each sampling period.

used in other fields, in order to change the time dynamics of the system. As a first order approximation, since the control variable is constant (the surface potential), the excursion of one or several state variables is reduced. The time response of the system no longer depends on its own free dynamics. It will depend on the marginal dynamics obtained within the control surface. This is extensively used, for example, in constant temperature anemometers to reduce their time response, [4].

\section{Closed-LOOP CONTROL}

The topology of the control loop is shown in Figure 1. It is a sampled system. At each sampling time the resistance of the nanostructured layer is measured. Depending on whether the measured value is above or below a threshold $\left(R_{t h}\right)$, either a BIT1 or BIT0 temperature waveform is applied during the next clock cycle (see Figure 2). Since both temperature waveforms end with the same temperature, $T_{\text {high }}$, the measurement of the resistivity is always made at the same temperature. The waveforms, BIT0 and BIT1, therefore respond to two needs:

- they allow to monitor the resistivity of the layer at constant temperature, $T_{\text {high }}$, and

- since they have two average temperatures, $\bar{T}_{\text {BIT0 }}<\bar{T}_{\text {BIT1 }}$, they allow to actuate on the surface potential. 


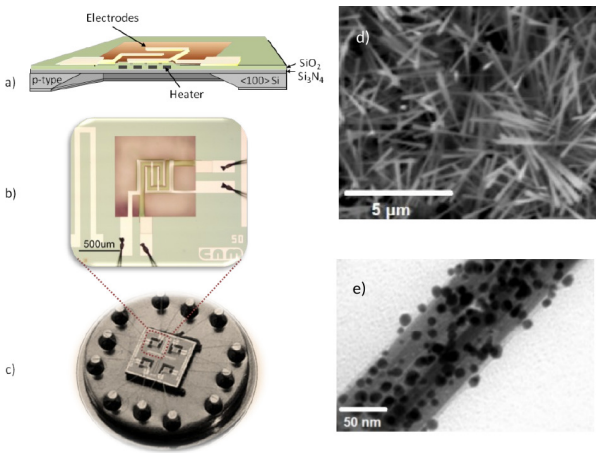

Figure 3. Drawing of a sensor (stack of interdigited Pt electrodes and polysilicon heating element sandwitched between silicon dioxide and silicon nitride layers (a); drawing of a sensor element seen from the top (b); a silicon chip comprises four micromachined sensors (c); SEM micrograph of CVDgrown tungsten oxide nanowires coating the electrode area (d); TEM image showing the $\mathrm{Au}$ nanoparticles coating a tungsten oxide nanowire.

The control loop has been designed to compensate changes of the surface potential by increasing (decreasing) the average temperature, if the resisitivity of the sensing layer was below (above) the desired value, $R_{t h}$.

\section{EXPERIMENTAL RESULTS}

The active layers used in this paper are made of nanoneedles of $\mathrm{WO}_{3}$ decorated with $\mathrm{Au}$ particles. The $\mathrm{WO}_{3}$ nanoneedles functionalized with gold have been grown in a single step using localized aerosol-assisted chemical vapor deposition (AACVD), of tungsten hexacarbonyl $\left(20 \mathrm{mg}, \mathrm{W}(\mathrm{CO})_{6}\right.$, Sigma-Aldrich, $\geq 97 \%$ ) with tetrachloroauric acid trihydrate ( $1 \mathrm{mg}, \mathrm{HAuCl}_{4} \cdot 3 \mathrm{H}_{2} \mathrm{O}$, Sigma-Aldrich, 99.9\%). These active sensing layers were grown on top of silicon MEMS membranes that comprise a pair of interdigitated gold electrodes and an embedded polysilicon resistor that performs the function of structure heater, [2]. Changes in temperature of the microstructure can be achieved in 10-50 ms.

The first experiment performed in air can be seen Figure 4. At the beginning of the experiment, the value of the resistance of the layer is approximately $250 \mathrm{k} \Omega$. The target value set by the closed-loop control in this case was $R_{t h}=450 \mathrm{k} \Omega$. During the first $35 \mathrm{~s}$ of the experiment the closed loop control only generates BIT1 waveforms, therefore increasing the surface potential of the nanostructures. After this initial phase, the resistance reaches the threshold value and the control begins to apply the necessary combination of BIT1 and BIT0 temperature waveforms (Figure 4.middle) to keep constant the average surface potential.

In a second experiment 14 target resistance values are set in the control loop in intervals of $120 \mathrm{~s}$. As it can be seen in Figure 5, the control achieves the target values set by the experiment by adequately changing the average temperature of the sensing layer.

\section{CONCLUSiONS}

The first experimental results of applying a closed-loop control to a nanostructured active layer made of $\mathrm{WO}_{3}$ nanoneedles functionalized with Au nanoparticles have been shown. The
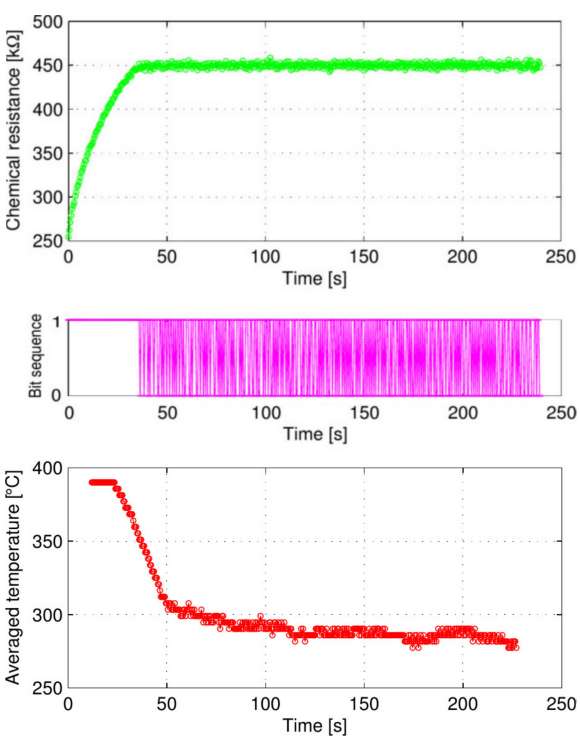

Figure 4. Experiment on which a target chemical resistance of $450 \mathrm{k} \Omega$ has been set as threshold value for the chemical control loop. Top: Plot of the chemical resistance as a function of time, measured at the reference temperature, $T_{\text {high }}$. Middle: sequence of BIT1 and BIT0 symbols applied to the device during the experiment. Bottom: Average temperature applied to the device (average of 50 temperature BIT0/BIT1 symbols each point). $T_{\text {high }}=415^{\circ} \mathrm{C}$ and $T_{\text {low }}=143^{\circ} \mathrm{C}$. Sampling period, $T_{S}=0.6 \mathrm{~s}, \delta=0.1$.
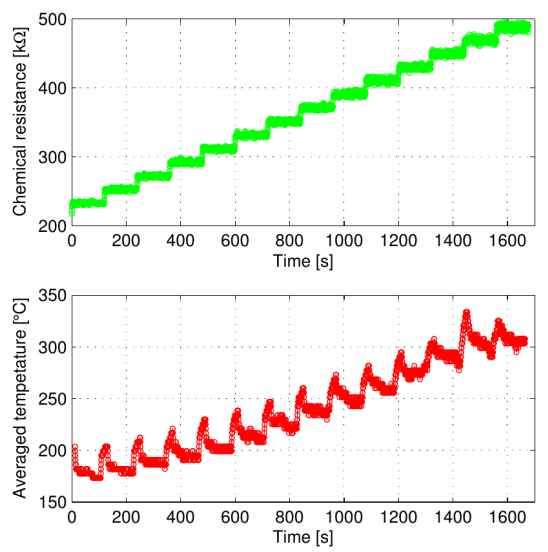

Figure 5. Top: Chemical resistance, measured at $T_{\text {high }}$, and at multiples of the sampling period, for an experiment on which the target resistance of the chemical loop is increased each 2 minutes from $230 \mathrm{k} \Omega$ in steps of $20 \mathrm{k} \Omega . T_{\text {high }}=415^{\circ} \mathrm{C}, T_{\mathrm{low}}=143^{\circ} \mathrm{C}, T_{S}=0.6 \mathrm{~s}, \delta=0.1$. Bottom: time evolution of the generated average temperature as a function of time.

proposed control loop is able to set arbitrary values of the average surface potential of the sensing layer.

\section{REFERENCES}

[1] M. C. McAlpine, H. Ahmad, D. Wang, and J. R. Heath, "Highly ordered nanowire arrays on plastic substrates for ultrasensitive flexible chemical sensors." Nat. Mater, vol. 6, no. 5, pp. 379-84, may 2007.

[2] F. Annanouch ; et al, "Aerosol-assisted CVD-grown WO3 nanoneedles decorated with copper oxide nanoparticles for the selective and humidityresilient detection of H2S." ACS Appl. Mater. Interfaces, vol. 7, no. 12, pp. $6842-51,2015$.

[3] F. Patolsky; et al, "Nanowire sensors for medicine and the life sciences." Nanomedicine (Lond)., vol. 1, no. 1, pp. 51-65, 2006.

[4] M. Dominguez; et al, "A hot film anemometer for the Martian atmosphere," Planet. Space Sci., vol. 56, no. 8, pp. 1169-1179, 2008. 